\title{
Peri-Operative Morbidity and Outcomes of Radical Cystectomy- Institutional Experience of Single Center
}

\author{
Amit Mani Upadhyay ${ }^{1}$, Ashok Kumar Kunwar ${ }^{1}$, Manik Lama ${ }^{1}$, Kabir Tiwari ${ }^{1}$, Sanjesh Bhakta Shrestha ${ }^{1}$, \\ Anil K. Shah ${ }^{1}$, Bishnu Dutta Poudel ${ }^{2}$, Kapendra Shekhar Amatya ${ }^{3}$ \\ ${ }^{1}$ Department of General Surgery, Phect-Nepal/Kathmandu Model Hospital, Kathmandu, Nepal \\ ${ }^{2}$ Department of Medical Oncology, NAMS, Bir Hospital, Kathmandu, Nepal \\ ${ }^{3}$ Department of Surgical Oncology, Nepal Cancer Hospital, Lalitpur, Nepal
}

\begin{abstract}
Introduction: The incidence of urinary bladder carcinoma increases distinctly with increasing age. Radical cystectomy has been the gold standard for the treatment of patients with muscle-invasive bladder cancer or recurrent high-grade non-muscle invasive bladder cancer. Our study aimed to see the peri-operative morbidity and surgical outcomes of the patient who had undergone radical cystectomy in our low volume center.

Methods: We retrospectively reviewed the inpatient charts as well as the outpatient records of 10 patients who had undergone radical cystectomy performed in our center for 9 years. A review of the literature on perioperative morbidity of radical cystectomy was also done using the combination of keywords like mortality, complications, and outcomes of surgery.

Results: Age of the patients ranged from 40-80 years. Eight of them were male and two were female. Painless hematuria (70\%) was the commonest presenting symptom, $80 \%$ of them were smokers. Three patients received neoadjuvant chemotherapy. Nine patients had radical cystectomy with an ileal conduit, whereas one patient had radical cystectomy with orthotopic neo-bladder.In postoperative complications, five patients had Clavien-Dindo grade I, three patients had grade II, one patient had grade IIIB and one patient had grade V complications. Two years of cancer-free survival was $90 \%$ and five years of cancer-free survival was $50 \%$.
\end{abstract}

Conclusions: Radical cystectomy with ileal conduit was still the choice of surgery in muscle-invasive and recurrent high-grade non-muscle invasive bladder cancer.

Keywords: Ileal Conduit, Muscle Invasive Bladder Cancer, Neo-bladder, Perioperative Morbidity, Radical Cystectomy.

\section{Introduction}

The incidence of urinary bladder carcinoma increases distinctly with increasing age. It is the second most common genitourinary malignancy after prostate cancer in men worldwide. The disease most commonly occurs beyond the age of 70 years of life.1 Radical cystectomy $(\mathrm{RC})$ remains the gold standard for treatment of patients with muscle-invasive bladder cancer or recurrent high-grade non-muscle invasive bladder cancer. ${ }^{2}$ Despite the advent of minimally invasive and robotic technology, radical cystectomy has significant mortality and morbidity. ${ }^{2} \mathrm{RC}$ is a technically challenging operation and hence prompt post-operative recovery, short hospital stay, and reduction in morbidity and mortality are difficult to achieve. ${ }^{1}$ The high rates of morbidity and mortality are because the majority of patients undergoing this procedure are elderly patients with multiple comorbidities. ${ }^{2}$

Correspondence

Dr. Amit Mani Upadhyay, Department of General Surgery, Phect-Nepal/Kathmandu Model Hospital, Exhibition Road, Kathmandu, Nepal, email: amitmaniupadhyay@gmail.com,ph: +977-9841281812 
In RC, morbidity is significant with a ninety-day complications rate between $28 \%$ to $64 \%$, even in high volume centers. ${ }^{3}$ Ninety-day mortality rates from different population studies range from $5.1 \%$ to $8.1 \%$, which are high for surgery with curative intent. 3 But the past two decades have witnessed several publications reporting the reduction in peri-operative mortality of $\mathrm{RC}$, reflecting the success of a multi-disciplinary approach. ${ }^{2}$ We aimed to evaluate the outcomes of radical cystectomy and its perioperative morbidity in our low volume center.

\section{Methods}

We retrospectively reviewed the inpatient charts as well as outpatient records of ten patients who had undergone Radical Cystectomy (RC) performed in Kathmandu Model Hospital from January 2011 to December 2019 for 9 years. A review of the literature on perioperative morbidity of radical cystectomy was also done using the combination of keywords like mortality, complications, and outcomes of surgery. Ethical clearance was obtained from the hospital's Institutional Review Committee(IRC) to conduct the study.

The diagnosis of the disease was confirmed by staging the Transurethral Resection of Bladder Tumor (TURBT) and cystoscopic biopsy. Staging of bladder cancer was done according to American Joint Committee on Cancer, TNM staging system. ${ }^{4}$ Perioperative complications were graded according to modified Clavien-Dindo classification. ${ }^{5}$ Complications which occurred after thirty days following $\mathrm{RC}$ were termed as late surgical complications.

\section{Results}

The age of patients ranged from 40-80 years, with a mean age of 61.9 years. Eight of them were male and two were female. Preoperative diagnoses of 9 patients were carcinoma of the urinary bladder and that of one was carcinoma of proximal urethra. The majority of them presented with painless hematuria (70\%). Eighty percent of them were smoker. Table 1 illustrates the demography and tumor characteristics in detail.

Preoperatively, $50 \%$ of them also had urine cytology positive for malignant cells. Five patients had muscleinvasive bladder cancer, three had recurrent multifocal T1 high-grade tumors, one had recurrent multifocal T1 low-grade tumors with posterior urethral stricture and one had urothelial carcinoma of proximal urethra. Out of ten patients, three patients who had locally advanced tumor received 3 cycles of neoadjuvant chemotherapy with Cisplatin and Gemcitabin for down-staging of the tumor (Figure 1).

Nine patients had undergone radical cystectomy with ileal conduit and orthotopic neo-bladder (Kock's ileal bladder substitution) was performed in one patient. Figure 2 illustrates the en bloc specimen removed during surgery. In eight patients, uretero-enteric anastomosis was completed by the Wallace technique, however, in two patients it was done by Bricker technique. Intraoperative blood loss ranged from $500-1000 \mathrm{ml}$ with a mean of $687 \mathrm{ml}$. One patient had transection of the left obturator nerve during pelvic lymph node dissection, which was repaired intraoperatively without postoperative sequel. The operative time varied from 240 minutes to 300 minutes with a mean of 262 minutes. The pelvic drain was removed within 7 to 10 days and conduit drain and ureteric stents were removed in 14 days on average. The average hospital stay of the patient varied from 17 to 25 days, with a mean hospital stay of 20.4 days.

On TNM staging, 5 patients had stage II disease, 3 had stage I, 1 had stage IIIA and 1 had stage IIIB disease respectively (Table1). Post-operative complications were categorized by the Modified Clavien-Dindo grading system. ${ }^{5}$ A majority had grade I (50\%) and grade II (30\%) complications followed by grade IIIB(10\%) and grade $\mathrm{V}(10 \%)$ complications(Table 2 ).

Table 1: Characteristics of the Patients $(\mathrm{N}=10)$

\begin{tabular}{|c|c|c|}
\hline \multicolumn{2}{|l|}{ Age (Years) } & $\begin{array}{l}\text { Number of } \\
\text { Patients (\%) }\end{array}$ \\
\hline \multicolumn{3}{|l|}{ Gender } \\
\hline \multicolumn{2}{|l|}{ Male } & $8(80 \%)$ \\
\hline \multicolumn{2}{|l|}{ Female } & $2(20 \%)$ \\
\hline \multicolumn{2}{|l|}{ Smoker } & $8(80 \%)$ \\
\hline \multicolumn{3}{|c|}{ Presenting Symptoms } \\
\hline \multicolumn{2}{|c|}{ Hematuria } & $7(70 \%)$ \\
\hline \multicolumn{2}{|c|}{ Irritative symptoms } & $3(30 \%)$ \\
\hline \multicolumn{3}{|c|}{ Types of Surgery } \\
\hline \multicolumn{2}{|c|}{ Radical Cystectomy with Ileal Conduit } & $9(90 \%)$ \\
\hline \multicolumn{2}{|c|}{ Radical Cystectomy with Neo-bladder } & $1(10 \%)$ \\
\hline \multicolumn{2}{|c|}{ Both neo-adjuvant and ad } & t $3(30 \%)$ \\
\hline \multicolumn{2}{|c|}{ Adjuvant Chemotherapy only } & $2(20 \%)$ \\
\hline \multicolumn{3}{|c|}{ TNM Staging } \\
\hline \multicolumn{3}{|c|}{ T1N0M0 } \\
\hline T2aN0M0 & Stage II & $2(20 \%)$ \\
\hline T2bN0M0 & Stage II & $3(30 \%)$ \\
\hline T2bN1M0 & Stage IIIA & $1(10 \%)$ \\
\hline T4aN2M0 & Stage IIIB & $1(10 \%)$ \\
\hline \multicolumn{3}{|c|}{ Cancer-free survival } \\
\hline \multicolumn{2}{|c|}{2 years survival } & $90 \%$ \\
\hline \multicolumn{2}{|c|}{5 years survival } & $50 \%$ \\
\hline
\end{tabular}


Table 2: Peri-operative and late surgical complications in the patients.

\begin{tabular}{|l|r|}
\hline List of early surgical complications & $\begin{array}{c}\text { No. of patients } \\
\text { (Percentage) }\end{array}$ \\
\hline Modified Clavien Dindo (Grade I) & $2(20 \%)$ \\
\hline Superficial wound infections & $2(20 \%)$ \\
\hline Lung atelectasis requiring physiotherapy & $1(10 \%)$ \\
\hline Thrombophlebitis & $2(20 \%)$ \\
\hline Modified Clavien Dindo (Grade II) & $1(10 \%)$ \\
\hline Blood transfusions \\
\hline Requiring TPN \\
\hline Modified Clavien Dindo (Grade IIIB) \\
\hline $\begin{array}{l}\text { Entero-cutaneous fistula requiring } \\
\text { exploration under general anesthesia }\end{array}$ \\
\hline Modified Clavien Dindo (Grade V) \\
\hline Patient death within 24 hours \\
\hline List of late surgical complications \\
\hline Subacute bowel obstruction & $1(10 \%)$ \\
\hline Pyelonphritis & $3(30 \%)$ \\
\hline Renal stones formation & $2(20 \%)$ \\
\hline Stomal prolapse & $2(20 \%)$ \\
\hline Parastomal hernia & $2(20 \%)$ \\
\hline Urinary incontinence & $1(10 \%)$ \\
\hline Disease Progression leading to mortality \\
\hline Liver metastasis & $2(20 \%)$ \\
\hline Rectal metastasis & $1(10 \%)$ \\
\hline
\end{tabular}

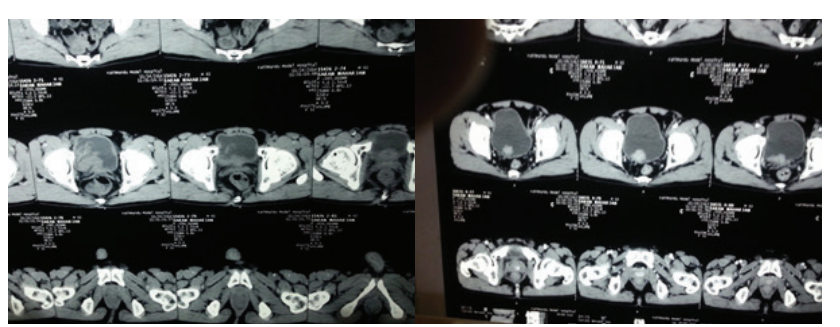

Figure 1: CT abdomen and pelvis shows large locally advanced urinary bladder tumor involving the base and right lateral wall of the bladder(left side) and a significant reduction in the size of tumor following neo-adjuvant chemotherapy(right side).

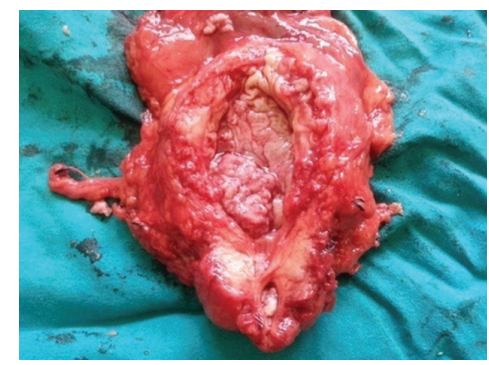

Figure 2: Radical cystectomy specimen of the same patient showing tumor in the base and right lateral wall of the urinary bladder.
Three patients developed recurrent subacute bowel obstruction; two patients had recurrent pyelonephritis which was managed non-operatively. One patient had mild stomal prolapse and one had significant stomal prolapse with a parastomal hernia which required further surgery. One patient who had undergone neo-bladder had urinary incontinence for the initial few months but he got continent by 6 months following RC. Nonobstructing renal stones were found in two patients, three years following the RC.Out of ten patients, six were advised for postoperative adjuvant chemotherapy; out of which one patient refused chemotherapy. So in total three patients received both neoadjuvant and adjuvant chemotherapy whereas two received adjuvant chemotherapy only.

All these patients were followed up according to the urinary bladder cancer treatment protocol. The average follow-up of the patients varied from 30 months to 78 months. Six patients were on regular follow up to date with no evidence of recurrence of the disease. Two years of cancer-free survival was $90 \%$, however, five-year cancer-free survival was found to be $50 \%$. One patient died within 24 hours following surgery and three patients died within three years following definitive surgery. Among three patients who died within three years after the surgery, two had liver metastasis and one had rectal metastasis despite receiving cisplatin-based adjuvant chemotherapy 4 to 6 cycles. These three patients refused further second-line chemo-radio therapy.

\section{Discussion}

Bladder cancer is among the top ten most common types of cancer in the world, with approximately 550,000 new cases annually. ${ }^{6}$ The incidence of urinary bladder carcinoma is four-fold higher in the male population and painless hematuria is the most common initial presenting symptom in the Nepalese population. Smoking is found to be one of the major risk factors in the development of bladder carcinoma. ${ }^{7}$ Transitional cell carcinoma(TCC) is the most common histological variety of bladder cancer in Nepal accounting more than $90 \%$ of cases. ${ }^{78}$

National Cancer Database reported 30 days and 90day postoperative mortality rates of $3.2 \%$ and $8.0 \%$ for radical cystectomy in low-volume centers and $2.7 \%$ and $7.2 \%$ in high-volume centers respectively. ${ }^{3}$ The enhanced recovery program (ERP) had been shown to reduce 
the occurrence of adverse events and length of stay for patients undergoing abdominal surgery across several disciplines. ${ }^{9}$

The impact of optimum surgical technique on the reduction of perioperative morbidity in RC depends on the following: (i) preoperative factors (ii) anesthesia and perioperative anesthetic management (iii) clinical care pathways (iv) operative Technique. ${ }^{2}$ In some highvolume institutions, the introduction of ERP had resulted in a reduction in the median length of hospital stay from 8 to 4 days. Carbohydrate loading before surgery had been recommended to minimize the development of insulin resistance and catabolism of protein and fat stores secondary to the physiological stress response seen in patients undergoing surgery. ${ }^{11}$ In our center, carbohydrate loading has been routinely practiced in major upper gastrointestinal, colorectal, and urological surgeries.

The majority of early complications following open radical cystectomy and urinary diversion occur as a direct result of the surgery and include gastrointestinal (29\%), infection (25\%), and wound-related complications (15\%). ${ }^{14}$ Postoperative ileus is regarded as the most frequent reason for a prolonged hospital stay following cystectomy. Early use of prokinetics such as metoclopramide had shown good results in reducing postoperative ileus in some of the studies. ${ }^{12,13}$ In our study, two cases had wound-related early complications (surgical site infections), one patient had entero-cutaneous fistula which needed exploration of the wound under general anesthesia and placement of a corrugated drain. The fistula healed spontaneously within two weeks. We observed prolonged ileus in a patient with orthotopic neobladder in comparison to an ileal conduit, though our case volume was very small to further elaborate this finding.

Radical cystectomy with ileal conduit or with orthotopic neo-bladder reconstruction has been considered as a challenging procedure that carries a significant risk of short and long term complications. ${ }^{10}$ Ileal substitution with freely refluxing uretero-ileal anastomosis is most commonly performed, although many techniques exist; not a particular technique has been considered superior. In our series, three patients had a recurrent subacute intestinal obstruction and two patients had recurrent pyelonephritis. These patients required multiple hospital admissions and were managed non-operatively. Two patients developed stomal prolapse and one had a parastomal hernia as well. Two patients also developed non obstructing renal stones as long term complication which did not require surgery. None of our patients developed calculus in ileal conduit or neobladder pouch. The patient with significant stomal prolapse and parastomal hernia underwent refashioning of the stoma and mesh repair of the parastomal hernia 3 years following RC.

Though radical cystectomy is the complex procedure and bears many serious perioperative complications, the complications are preventable by some suggested measures by different authors. ${ }^{15,16} \mathrm{Technical}$ advancement in the surgical and anesthesia practices, multidisciplinary approach for correction and control of co-morbidities and early postoperative rehabilitation have produced a satisfactory effect in reducing the mortality. ${ }^{17}$

All our patients received epidural analgesia for postoperative pain management which decreased the need for opioids significantly and early chest physiotherapy also helped us in decreasing lung-related complications. Talking about the operative technique, after encountering entero-cutaneous fistula in one patient, we started doing reinforcement of the small bowel serosa layer with 3-0 vicryl after doing ileo-ileal anastomosis with a surgical stapler. We did not encounter anastomosis related complications thereafter. In our study, we lost one patient within 24 hours of surgery due to severe cardiac arrhythmia which resulted in asystole. Our patient was a chronic smoker with a history of chronic obstructive pulmonary disease(COPD) and she had received three cycles of cisplatin-based neoadjuvant chemotherapy for down-staging of the tumor, which might have caused some degree of cardiac toxicity as well which was not evident preoperatively.

In another study, a significant proportion (15.6\%) of complications occurred after discharge from the hospital, most notably infectious complications, deep vein thrombosis(DVT), and fascial dehiscence. ${ }^{19}$ In a similar study by Surveillance, Epidemiology, and End Results (SEER)-Medicare data showed that as many as one-quarter of patients required readmission after cystectomy with $33.8 \%$ of those having complications. 18 Surprisingly, none of our patients developed DVT or fascial dehiscence within three months following the surgery. Our two patients developed wound-related 
SSI which was managed before the discharge from the hospital and two patients got readmitted due to pyelonephritis within three months following the RC and were treated non-operatively.

Despite many efforts to improve radical cystectomy perioperative care which includes refinement of surgical technique, technological advances, and thrombotic/ infectious prophylaxis; postoperative complications remain very high. ${ }^{19}$ Improved outcomes are directly related to proper patient selection. Many studies have identified factors that are associated with poor outcomes of surgery such as increased age, female gender, comorbidities, previous radio/chemotherapy, low serum albumin concentration, and longer duration of surgery. ${ }^{20 \text {, }}$ ${ }^{23}$ The result of our small group of studies is also similar to the results of the studies described above. We encountered mortality in a lady with COPD who also had received neoadjuvant chemotherapy and the patient who developed entero-cutaneous fistula, was more than 70 years of age who also had concomitant diabetes mellitus and hypertension.

As already described above, it is obvious that due to the older age of the patient and co-morbidities, many risk factors cannot be modified, however, preoperative identification of a high-risk patient can help the treating clinicians to modify their approach and ensure proper patient counseling and monitoring. ${ }^{24}$ Some interventions have been shown to decrease surgical complications including use of electrosurgical heat-sealing devices that reduce intra-operative blood loss, use of surgical staplers in small bowel resection and anastomosis which reduces operative time and adherence to proper antibiotic and deep vein thrombosis prophylaxis guidelines. ${ }^{20,21}$

We did not routinely use thrombosis prophylaxis in our patients. However, we used a heat sealing device in one of our patients undergoing RC but due to a low number of cases, it was difficult to compare the amount of intra-operative bleeding with conventional coagulation techniques. However, after the use of surgical stapler for ileo-ileal anastomosis, our operative time decreased by almost thirty minutes on average.

Physicians need to be aware of the proper use of their disposal and ensure they were applied consistently in all cystectomy patients given their high risk of adverse outcomes. ${ }^{22}$ It is understood that age more than 70 years and female patients have been labeled as higher risk factors with the poor postoperative outcome of RC.24 However, with proper selection and risk factors balancing, there is a beneficial impact on the reduction in morbidity.

Renal failure (serum creatinine $>2.5 / \mathrm{dl}$ ) and poor nutritional status require preoperative correction wherever possible to reduce complications. ${ }^{25}$ Generally, elderly patients who also have derangement of renal function, urinary diversion with an ileal conduit is preferable over complex procedures like orthotopic bladder substitution. ${ }^{26}$ None of our patients had preoperative derangement of renal function but two patients developed persistently high serum creatinine level postoperatively but in both cases, serum creatinine was within $2 \mathrm{mg} / \mathrm{dl}$ which did not require subsequent treatment.

Few other studies also suggested that patients undergoing RC after neoadjuvant chemotherapy had a significant risk of perioperative morbidity. ${ }^{27,28}$ Also, in our study bleeding tendency was found higher in patients following neoadjuvant chemotherapy which required blood transfusion intra-operatively as well as post-operatively. In our patients, intraoperative blood loss ranged from $500-1000 \mathrm{ml}$ with a mean of $687 \mathrm{ml}$.

Neo-adjuvant chemo-radiotherapy has been the mainstay of the treatment of bulky or loco-regionally advanced bladder tumor for the down-staging of the tumor. ${ }^{29}$ However, the patient undergoing RC following neo-adjuvant chemo or radiotherapy might lead to some technical difficulties during surgery and postoperative period due to adhesions and bleeding tendency. ${ }^{30,31} \mathrm{But}$ with careful planning and use of modern techniques, those complications could be minimized. ${ }^{20,21}$ In our study also three patients who had locally advanced tumor received neoadjuvant chemotherapy for downstaging of the tumor (Figure1).

Older patients with significant co-morbidities are better suited for the ileal conduit. In our experience also, the ileal conduit was better accepted by the patient as it is easy to care for the stoma by the patient with very good quality of life and minimum pouch related complications. But in the younger age group, orthotopic neo-bladder will be still highly preferable over ileal conduit by the patient.

\section{Conclusion}

In our setup with limited resources, radical cystectomy with ileal conduit was the choice of surgery for the 
management of muscle-invasive and high-grade superficial bladder cancer. It was technically easier, less time consuming, and can be performed with an acceptable rate of early complications even in low volume centers like our institution.

\section{Acknowledgment}

Professor Dr. Ganesh Dangal for reviewing and editing the manuscript.

Conflicts of Interest: None

Financial Support: Nil

\section{References}

1. Ferlay J, Autier P, Boniol M, Colombet M, Boyle P. Estimates of the Cancer incidence and mortality in Europe in 2006. Ann Oncol.2007; 18:581-92.

2. Kulkarni JN. Perioperative morbidity of radical cystectomy: A review. Journal of the Urological Society of India.2011; 27(2):226-32.

3. Tan WS, Lamb BW and Kelly J. Complications of Radical Cystectomy and Orthotopic Reconstruction. Adv Urol. 2015:323157.

4. Edge SB, Compton DR, Fritz CC, Greene AG, Trotti FL. AJCC cancer staging manual. 7thed. New York: Springer; 2010.

5. Dindo D, Demartines $\mathrm{N}$ and Clavien PA. Classification of Surgical Complications: A New Proposal with Evaluation in a Cohort of 6336 Patients and Results of a Survey. Ann Surg. 2004; 240(2):205-13.

6. Richters A, Aben KKH, Kiemeney LALM.The global burden of urinary bladder cancer: an update. World Journal of Urology. 2019:31676912. DOI: 10.1007/s00345-019-02984-4

7. Joshi HN, Makaju R, Karmacharya A, Karmacharya RM, Shrestha B, Shrestha R. Urinary Bladder Carcinoma: Impact of Smoking, Age and its ClinicoPathological Spectrum. Kathmandu University Medical Journal.2013; 11(4): 44.

8. Vaidya S,Lakhey $M, \quad K C S$, Hirachand S. Urothelial Tumours of the Urinary Bladder: A Histopathological Study of Cystoscopic Biopsies.J Nepal Med Assoc. 2013;52(191):475-8.

9. Kehlet H. Multimodal approach to control postoperative pathophysiology and rehabilitation. British Journal of Anaesthesia.1997; 78(5):606-17.

10. Daneshmand S, Ahmadi H,Schuckmanetal AK. Enhanced recovery protocol after radical cystectomy for bladder cancer.The Journal of Urology. 2014; 192(1):50-5.

11. Thorell A, Nygren J, Ljungqvist O. Insulin resistance: a marker of surgical stress.Current Opinion in Clinical Nutrition and Metabolic Care.1999; 2(1):69-78.

12. Chang SS, Baumgartner RG, Wells N, Cookson MS,Smith JA. Causes of increased hospital stay after radical cystectomy in a clinical pathway setting. J Urol.2002; 167(1):208-11.

13. Lee CT, Chang SS, Kamat AM. Alvimopan accelerates gastrointestinal recovery after radical cystectomy: a multicenter randomized placebocontrolled trial. Eur Urol. 2014; 66(2):265-72.

14. Shabsigh A, Korets R, Vora KC. Defining early morbidity of radical cystectomy for patients with bladder cancer using a standardized reporting methodology. Eur Urol. 2009; 55(1):164-74.

15. Thomas DM, Riddle PR. Morbidity and mortality in 100 consecutive radical cystectomies. Br J Urol. 1982;54:716-9.

16. Sullivan JW, Grabstald H, Whitmore WFJr. Complications of ureteroileal conduit with radical cystectomy: review of 336 cases. J Urol. 1980;124:797-801.

17. Donat SM. Standards for surgical complication reporting in urologic oncology: time for a change. Urology. 2007;69:221-5.

18. Hu M, Jacobs BL, Montgomery JS, He C, Ye J. Sharpening the focus on causes and timing of readmission after radical cystectomy for bladder cancer. Cancer. 2014; 120(9):1409-16.

19. Lavallée LT, Schramm D, Witiuk K, Mallick R, Fergusson D, Morash C. Peri-Operative Morbidity Associated with Radical Cystectomy in a Multicenter Database of Community and Academic Hospitals. PLoS ONE. 9(10): e111281.

20. Thompson IM, 3rd, Kappa SF, Morgan TM, Barocas DA, Bischoff CJ. Blood loss associated with radical cystectomy: A prospective, randomized study comparing impact LigaSure vs. stapling device.Urol Oncol. 2014; 32(1):45.e11-5.

21. Karl A, Buchner A, Becker A, Staehler M, Seitz M. A new concept for early recovery after surgery for patients undergoing radical cystectomy for bladder cancer: Results of a prospective randomized study. J Urol. 2014; 191(2):335-40.

22. Cerantola Y, Valerio M, Persson B, Jichlinski P, 
Ljungqvist O. Guidelines for perioperative care after radical cystectomy for bladder cancer: Enhanced recovery after surgery (ERAS) society recommendations. ClinNutr. 2013; 32:879-87.

23. Lee KL, Freiha F, Presti JCJr, Gill HS. Gender differences in radical cystectomy: complications and blood loss. Urology. 2004;63:1095-9.

24. Gamé X, Soulié M, Seguin P, Vazzoler N, Tollon C, Pontonnier F, Plante P. Radical cystectomy in patients older than 75 years: assessment of morbidity and mortality. Eur Urol. 2001;39:525-9.

25 Siddiqui K M. and Izawa JI. Ileal conduit: standard urinary diversion for elderly patients undergoing radical cystectomy. World Journal of Urology.2016; 34: 19-24.

26. Young $M J$, Elmussareh $M$, Weston $P$ and Dooldeniya $M$. Radical cystectomy in the elderly - Is this a safe treatment option? Arab J Urol. 2017; 15(4): 360365.

27. Khochikar MV.Treatment of locally advanced and metastatic bladder cancer. Indian J Urol. 2008;
24(1): 84-94.

28. Nagele U, Anastasiadis AG, Merseburger AS, Corvin S, Hennenlotter J, Adam M. The rationale for radical cystectomy as primary therapy for T4 bladder cancer. World J Urol.2007;25:401-5.

29. Marcq G, Jarry E, Ouzaid I, Hermieu JF, Henon F, Fantoni JC and Xylinas E. Contemporary best practice in the use of neoadjuvant chemotherapy in muscle-invasive bladder cancer. Ther Adv Urol. 2019; 11: 1756287218823678.

30. Manoharan M, Reyes MA, Kava BR, Singal R, Kim SS, Soloway MS. Is adjuvant chemotherapy for bladder cancer safer in patients with an ileal conduit than a neobladder? Br J Urol Int. 2005;96:1286-9.

31. Donat SM, Shabsigh A, Savage C, Cronin AM, Bochner BH, Dalbagni G. Potential impact of postoperative early complications on the timing of adjuvant chemotherapy in patients undergoing radical cystectomy: a high-volume tertiary cancer center experience. Eur Urol. 2009;55:177-86. 